\title{
A role for heterologous gap junctions between melanoma and endothelial cells in metastasis
}

\author{
Akihiko Ito, ${ }^{1}$ Fumitaka Katoh, ${ }^{2}$ Tatsuki R. Kataoka, ${ }^{1}$ Morihito Okada, ${ }^{3}$ \\ Noriaki Tsubota, ${ }^{4}$ Hideo Asada, ${ }^{5}$ Kunihiko Yoshikawa, ${ }^{5}$ Sakan Maeda, ${ }^{6}$ \\ Yukihiko Kitamura, ${ }^{1}$ Hiroshi Yamasaki, ${ }^{7}$ and Hiroshi Nojima ${ }^{8}$ \\ ${ }^{1}$ Department of Pathology, Medical School, Osaka University, Suita, Japan \\ ${ }^{2} \mathrm{Nippon}$ Shinyaku, Kyoto, Japan \\ ${ }^{3}$ Second Department of Surgery, Kobe University Medical School, Kobe, Japan \\ ${ }^{4}$ Department of Thoracic Surgery, Hyogo Medical Center for Adults, Akashi, Japan \\ ${ }^{5}$ Department of Dermatology, Medical School, Osaka University, Suita, Japan \\ ${ }^{6}$ Second Department of Pathology, Kobe University Medical School, Kobe, Japan \\ ${ }^{7}$ Unit of Multistage Carcinogenesis, International Agency for Research on Cancer, Lyon, France \\ ${ }^{8}$ Department of Molecular Genetics, Research Institute for Microbial Diseases, Osaka University, Suita, Japan
}

Address correspondence to: Hiroshi Nojima, Department of Molecular Genetics, Research Institute for Microbial Diseases, Osaka University, Yamada-oka 3-1, Suita 565-0871, Japan. Phone: 81-6-6875-3980;

Fax: 81-6-6875-5192; E-mail: hnojima@biken.osaka-u.ac.jp.

Received for publication August 25, 1999, and accepted in revised form March 13, 2000.

F10 and BL6 sublines of B16 mouse melanoma cells are metastatic after intravenous injection, but only BL6 cells are metastatic after subcutaneous injection. We found that connexin (Cx) 26 is upregulated in BL6 cells. To examine gap junction formation, we devised a coculture system, in which an opened vein segment was placed at the bottom of a culture dish and then dye-labeled melanoma cells were seeded onto it. Immunohistochemistry indicated that the vein segment preserved the integrity of the endothelial monolayer. In this system, BL6 cells could transfer dye into endothelial cells but F10 cells could not. Transfection with wild-type Cx26 rendered F10 cells competent for coupling with endothelial cells and as spontaneously metastatic as BL6 cells. Conversely, transfection with a dominant-negative form of Cx26 rendered BL6 cells deficient in coupling and less metastatic. In human melanoma lesions, the level of $\mathrm{Cx} 26$ expression was low in melanoma cells residing in the basal layer, but significantly upregulated in melanoma cells invading the dermis. The results suggested that $\mathrm{Cx} 26$ plays a role in intravasation and extravasation of tumor cells through heterologous gap junction formation with endothelial cells.

J. Clin. Invest. 105:1189-1197 (2000).

\section{Introduction}

Among B16 mouse melanoma cells, F10 and BL6 cells are nearly identical except for their metastatic behavior (1). Both cell types colonize the lungs when injected into the tail vein (experimental metastasis assay). In contrast, when injected subcutaneously into the footpad (spontaneous metastasis assay), BL6 cells are much more metastatic than F10 cells (1). Since the spontaneous metastasis assay reflects the in vivo sequence of metastatic events, it appears that BL6 cells may possess the full complement of factors essential for metastasis, all or some of which may be missing from F10 cells. Alternatively, F10, but not BL6 cells, may express a factor that suppresses metastasis. Genes encoding such factors are likely to be differentially expressed between the two cell lines. We have reported previously an improved method for constructing subtracted cDNA libraries (2-4). Application of this method to F10 and BL6 cells yielded a set of genes that were transcriptionally upregulated in BL6 cells $(5,6)$. Here, we report that connexin $26(\mathrm{Cx} 26)$ is one of these genes and may regulate the metastatic phenotype of melanoma cells.
Connexins are a family of at least 12 proteins that make up the intercellular channels of gap junctions (7). Gap junction hemichannels, called connexons, are composed of homomeric or heteromeric hexamers of connexins and provide conduits for the direct passage of ions and other low-molecular-weight molecules, including second messengers, between cells (8). This type of intercellular communication is the essence of direct contact-mediated cell-growth control and is important for many physiologic and developmental processes (9-11). Several reports have shown that gap junctional intercellular communication (GJIC) is disturbed during carcinogenesis (12-16). On the other hand, the role of connexins in the acquisition of more malignant phenotypes remains controversial (17-20).

Cx26 expression has been examined intensively in hepatocytes and squamous epithelial cells under physiologic and pathologic conditions (15, 21-24). The expression level of $\mathrm{Cx} 26$ is believed to decrease as hepatocytes and keratinocytes acquire the potential to proliferate autonomously $(21,23)$. We reported that transfection with $\mathrm{Cx} 26$, but not with $\mathrm{Cx} 40$ and $\mathrm{Cx} 43$, 
produced an inhibitory effect on the in vitro and in vivo growth of HeLa squamous cell carcinoma cells (15). On the other hand, the role of $\mathrm{Cx} 26$ during carcinogenesis of melanocytes and the malignant progression of melanoma cells is poorly understood. In the present study, we found that BL6 cells expressed Cx26 as abundantly as liver tissues. Subsequently, BL6 cells, but not F10 cells, were found to constitute a GJIC-competent cell population. Interestingly, BL6 cells could readily form a heterologous gap junction with endothelial cells, but could not form them efficiently with each other. The spontaneous metastasis assay indicated that the formation of such heterologous gap junctions by Cx26 might be responsible for the successful metastasis of F10 and BL6 cells.

\section{Methods}

Cell lines and culture. B16, F10, and BL6 melanoma cells were kindly provided by I.J. Fidler (The University of Texas, Houston, Texas, USA). NIH3T3 and SVEC4-10 mouse endothelial cells were purchased from American Type Culture Collection (ATCC; Manassas, Virginia, USA). All cells were maintained in DMEM supplemented with $10 \%$ fetal calf serum (growth medium). HUV-ECC human endothelial cells were purchased from ATCC and maintained in HUVE cell culture medium (Nissui Pharmaceutical Co., Tokyo, Japan). To maintain the transfectants, the growth medium was supplemented with G-418 at a final concentration of $2 \mathrm{mg} / \mathrm{mL}$.

Isolation of cDNA clones. Total RNA was extracted by the guanidine thiocyanate/CsTFA method from F10 and BL 6 cells, and then poly $(A)^{+}$RNA was purified by using oligo-dT cellulose. The cDNA libraries were prepared by the linker-primer method using plasmid pAP3neo, and the subtracted cDNA library was constructed according to methods described elsewhere (2-4). After the subtraction process was repeated four times, approximately 1,500 clones were rescued. From these, the clones transcriptionally upregulated in BL6 cells were selected by Northern blot analysis and sequenced. The DNA sequences were used to search the high throughput genomic sequences (htgs) database (http://www.ncbi.nlm.nih.gov/BLAST/blast_databases.html) using the Basic Alignment Search Tool (BLASTN) algorithm. Colonies $\left(2 \times 10^{6}\right)$ of the BL6 cDNA library in pAP3neo were transferred to a nylon membrane and hybridized with $\left[\alpha-{ }^{32} \mathrm{P}\right] \mathrm{dCTP}$-labeled cDNA inserts of clones from the subtracted cDNA library. Plasmid DNAs were purified from the positive colonies and sequenced.

Plasmid construction. Using the PAP3neo vector containing the full-length cDNA for $\mathrm{Cx} 26$ (pAP3neo-Cx26) as a template, PCR was performed with the following set of primers: F-Asc-ATG, 5'-CATGGCGCGCCGATGGATTGGGGCACACTCC-3' (containing an AscI site at the $5^{\prime}$ end); R-Cx26, 5'-TTTGTTTGGGAGCTTTGGGG-3'. The PCR-amplified DNA fragments, corresponding to the $5^{\prime}$ portion of Cx26 cDNA, were treated with Klenow DNA polymerase and then with BstXI. The plasmid con- struct pAP3neo-Cx26 was sequentially treated with $M l u I$, Klenow DNA polymerase, and BstXI to obtain the linearized plasmid construct lacking the $5^{\prime}$ portion of the Cx26 cDNA. The resulting plasmid was ligated with the PCR-amplified DNA fragments treated to contain blunt $5^{\prime}$ ends and BstXI sites at the $3^{\prime}$ ends. The $1.3-\mathrm{kb}-$ long, in-frame AscI-ApaI (the ApaI site is located in the 3' UTR of Cx26) cDNA fragment for Cx26 was used as an insert for ligation with the pcDNA3 expression vector, which contains the cytomegalovirus promoter (Invitrogen, San Diego, California, USA), after modifying pcDNA3 in the following manner (pcDNA3$\left.\mathrm{Cx} 26^{\mathrm{WT}}\right)$. A synthetic linker carrying an AscI site (5'AGCTTGGCGCGCCA-3') was inserted into the HindIII site of the pcDNA3 vector. This modification allowed the cDNA insert to be fused in-frame by way of the AscIApaI sites. Three substitutions of Cys- $60 \rightarrow$ Phe, Pro- 87 $\rightarrow$ Leu, and Arg-143 $\rightarrow$ Trp were introduced into the wild-type cDNA of Cx26 using the site-directed mutagenesis method as described (pcDNA3-Cx26 $660 \mathrm{~F}$, pcDNA3-Cx26 $6^{\mathrm{P} 87 \mathrm{~L}}$, and pcDNA3-Cx26 $6^{\mathrm{R} 143 \mathrm{~W}}$ ) (25). To obtain the mouse full-length cDNA for Cx32, PCR was performed using the mouse liver $\mathrm{CDNA}$ as a template with the following set of primers: F-Cx32, 5'-CATGGCGCGCCGATGAACTGGACAG-3' (containing an AscI site at the $5^{\prime}$ end); R-Cx32, 5'-TAGGAAGGGAGGGAAGGTTTGA-3'. The PCR-amplified cDNA fragment was subcloned into the T7Blue vector (Novagen, Madison, Wisconsin, USA). After amplification, the 1.0-kb-long, in-frame AscI-NotI (the NotI site is located in the multicloning site of the T7Blue vector) cDNA fragment for Cx32 was excised and ligated with the modified pcDNA3 vector by way of AscI-NotI sites (pcDNA3$\mathrm{C} \times 32^{\mathrm{WT}}$ ). All the plasmid constructs were sequenced to confirm that no mutation had been introduced by PCR.

Transfectant clones. F10 and BL6 cells were transfected with the pcDNA3 vector containing the wildtype or mutated forms of $\mathrm{Cx} 26$ or Cx32 using the calcium phosphate coprecipitation technique and selected for resistance to G-418 $(2 \mathrm{mg} / \mathrm{mL})$. The F10 clones were: F10-pcDNA3 containing the empty pcDNA3; three independent clones, F10-Cx $26^{\mathrm{WT}}-1$, 2 , and -3 containing pcDNA3-Cx $26^{\mathrm{WT}}$; F $10-\mathrm{C} \times 26^{\mathrm{C} 60 \mathrm{~F}}$, F10-Cx26 $6^{\mathrm{P} 87 \mathrm{~L}}$, and $\mathrm{F} 10-\mathrm{Cx} 26^{\mathrm{R} 143 \mathrm{~W}}$ containing pcDNA3-Cx26 ${ }^{\mathrm{C} 60 \mathrm{~F}}, \mathrm{pcDNA} 3 \mathrm{Cx} 26^{\mathrm{P} 87 \mathrm{~L}}$, and pcDNA3Cx26 $6^{\mathrm{R} 143 \mathrm{~W}}$, respectively; two independent clones, F10-Cx $32^{\mathrm{WT}}-1$ and -2 containing pcDNA3-Cx32 ${ }^{\mathrm{WT}}$. BL6 clones were: BL6-pcDNA3 containing the empty pcDNA3; two independent clones, BL6-Cx $26^{\mathrm{C} 60 \mathrm{~F}}-1$ and -2 containing pcDNA3-Cx $26^{\mathrm{C} 60 \mathrm{~F}}$; and three independent clones, BL6-Cx26 $6^{\mathrm{R} 143 \mathrm{~W}}-1,-2$, and -3 containing PcDNA3-Cx26 $6^{\mathrm{R} 143 \mathrm{~W}}$.

Northern blot analysis. Five micrograms of total RNA extracted from cultured cells, mouse tissues, or in vivo growing tumors were fractionated on $1 \%$ agaroseformaldehyde gels, transferred to a nylon membrane, and hybridized to DNA probes labeled with $\left[\alpha-{ }^{32} \mathrm{P}\right] \mathrm{dCTP}$ by the random hexamer labeling method. The Cx26 probe was prepared from the AscI-ApaI cDNA fragment 
encompassing the open reading frame. The probes of Cx32 (nucleotides [nt] 50-582) (26), Cx37 (nt 73-940) (27), Cx40 (nt 154-826) (28), and Cx43 (nt 458-1075) (GenBank accession number X61576) were obtained using PCR. The $\beta$-actin probe was prepared in the same fashion (3). The blots were washed to a final stringency of $0.1 \times \mathrm{SSC}$ and $0.1 \% \mathrm{SDS}$ at $50^{\circ} \mathrm{C}$ before exposure. Relative signal intensity was calculated with the BAS 2000 system (Fuji Photo Film Co., Tokyo, Japan).

Western blot analysis. Cell lysates were prepared by alkaline treatment to enrich for integral membrane proteins according to the procedure as described (29). One microgram of cell lysate proteins was separated by $10 \%$ SDS-PAGE and transferred onto an Immobilon membrane (Millipore Corp., Bedford, Massachusetts, USA). The membrane was incubated with mouse anti-Cx26 $\mathrm{mAb}$ (1:300 dilution) (CX-12H10; Zymed, San Francisco, California, USA) for 2 hours and then incubated with horseradish peroxidase-conjugated anti-mouse IgG antibody (1:1000 dilution; Cappel Research Products, Durham, North Carolina, USA). After washing with PBS, the blot was reacted with Renaissance chemiluminescence reagents (NEN Life Science Products Inc., Boston, Massachusetts, USA) before exposure. A duplicate gel was stained with silver according to the manufacturer's instructions (Daiichi Pure Chemicals Co., Tokyo, Japan).

Preparation and culture of vein segments. The tissue preparation was done essentially according to the procedure as described (30). Vein segments (measuring approximately $8 \mathrm{~mm}$ ) were dissected carefully from the thoracic portion of inferior vena cava (IVC) of freshly sacrificed mice and immediately transferred into growth medium. Afterward, a thin sonde was inserted into the lumen of the vein, and a longitudinal cut was made by a scalpel blade on the vein wall along with the sonde. The vein segment, with its luminal surface uppermost, was carefully stretched and pinned to a sheet of silicone rubber using minute pins (Watkins \& Doncaster, Kent, United Kingdom). As soon as the segment was fixed, the rubber sheet with the vein was placed on the bottom of a $35-\mathrm{mm}$ culture dish filled with growth medium. The area of the opened vein was approximately $7 \mathrm{~mm} \times 7 \mathrm{~mm}$ square. After the preparation above, the dye-transfer assay was performed using the vein culture. Some segments of vein were used to visually monitor endothelial integrity. At 0,2 , 4 , and 6 hours after the initiation of the culture, vein segments were incubated for 1-2 minutes at room temperature in PBS containing 0.01\% trypan blue (Flow Laboratories, Irvine, Scotland), and observed through a stereomicroscope (SZH-ILLD; Olympus Optical Co., Osaka, Japan).

Dye-transfer assay. To label melanoma cells, 2'7'bis(carboxyethyl)-5(and-6)carboxyfluorescein (BCECF) acetoxymethyl ester (Dojindo, Kumamoto, Japan) was added to a log-phase culture at a final concentration of $1 \mu \mathrm{M}$. Three hours later, the medium was changed and cultivation was continued for 1 hour. Cells were
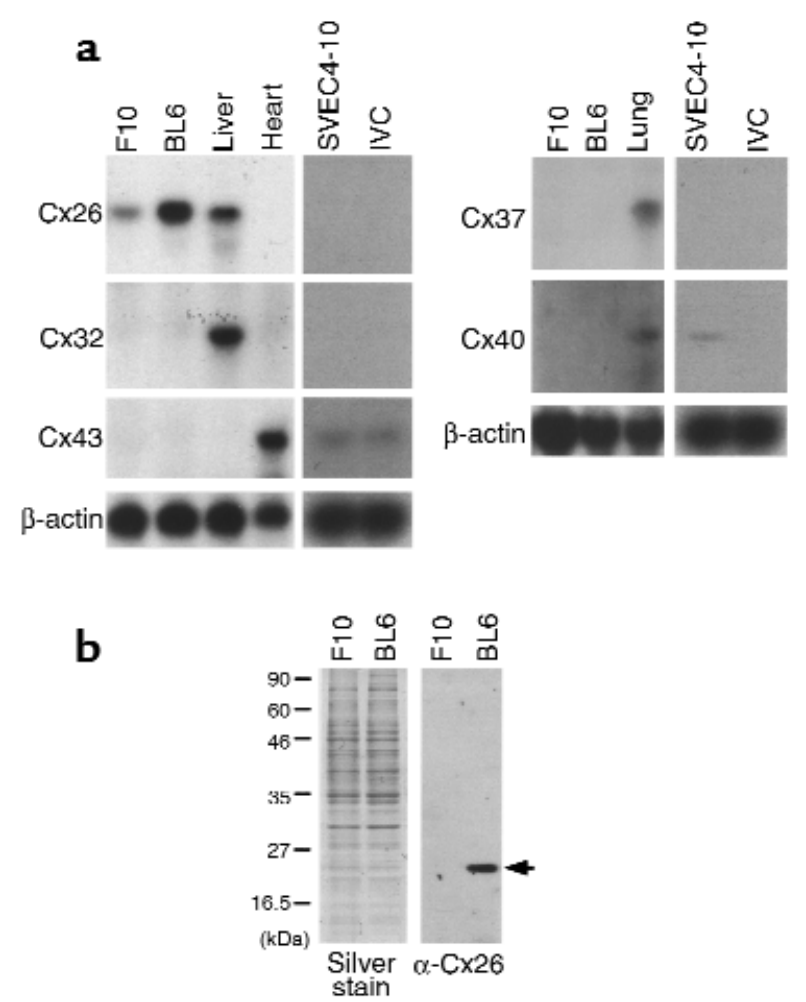

Figure 1

Expression of various connexins in F10 and BL6 cells and in the IVC segment. (a) Northern blot analysis to detect the transcripts of $\mathrm{C} \times 26$, $\mathrm{C} \times 32, \mathrm{C} \times 43, \mathrm{C} \times 37$, and $\mathrm{C} \times 40$. As a positive control, mouse liver, heart and lung tissues, and SVEC4-10 cells were used. (b) Expression of Cx26 protein in F10 and BL6 cells. Cell lysates were extracted by alkaline treatment. Anti-Cx26 antibody detected monomeric $\mathrm{C} \times 26$ protein at approximately $24 \mathrm{kDa}$ (indicated by an arrow in the right panel). Equal protein loading was confirmed by silver staining of the gel (left panel).

harvested and washed three times with PBS. Almost all the cells displayed equal fluorescent intensity. In vitro GJIC formation between melanoma cells, between melanoma and NIH3T3 cells, between melanoma and SVEC4-10 cells, and between melanoma and HUV-EC$\mathrm{C}$ cells was estimated using the monolayer cell culture as described (15). To prepare the coculture of melanoma cells and a vein segment, BCECF-labeled cells $\left(10^{6}\right)$ were seeded on the vein segment that had been placed on the bottom of a $35-\mathrm{mm}$ culture dish. At various time points, the cocultured tissue was fixed with $3 \%$ glutaraldehyde in phosphate buffer $(0.1 \mathrm{M}$, pH 7.2) and observed directly through an Axioplan2 microscope (Carl Zeiss, OberKochen, Germany) equipped for epifluorescence. The fluorescent intensity was converted into 256-color spectrum by Adobe Photoshop (Ver 3.5, Adobe Systems Inc., Mountain View, California, USA) software on a Macintosh computer. The number of cells showing dye coupling with endothelial cells was calculated by observing 500 cells per dish and pooling the data from three experiments.

Immunohistochemistry. Vein segments at various intervals of cultivation and human melanoma tissues were fixed with $4 \%$ paraformaldehyde, embedded in paraf- 


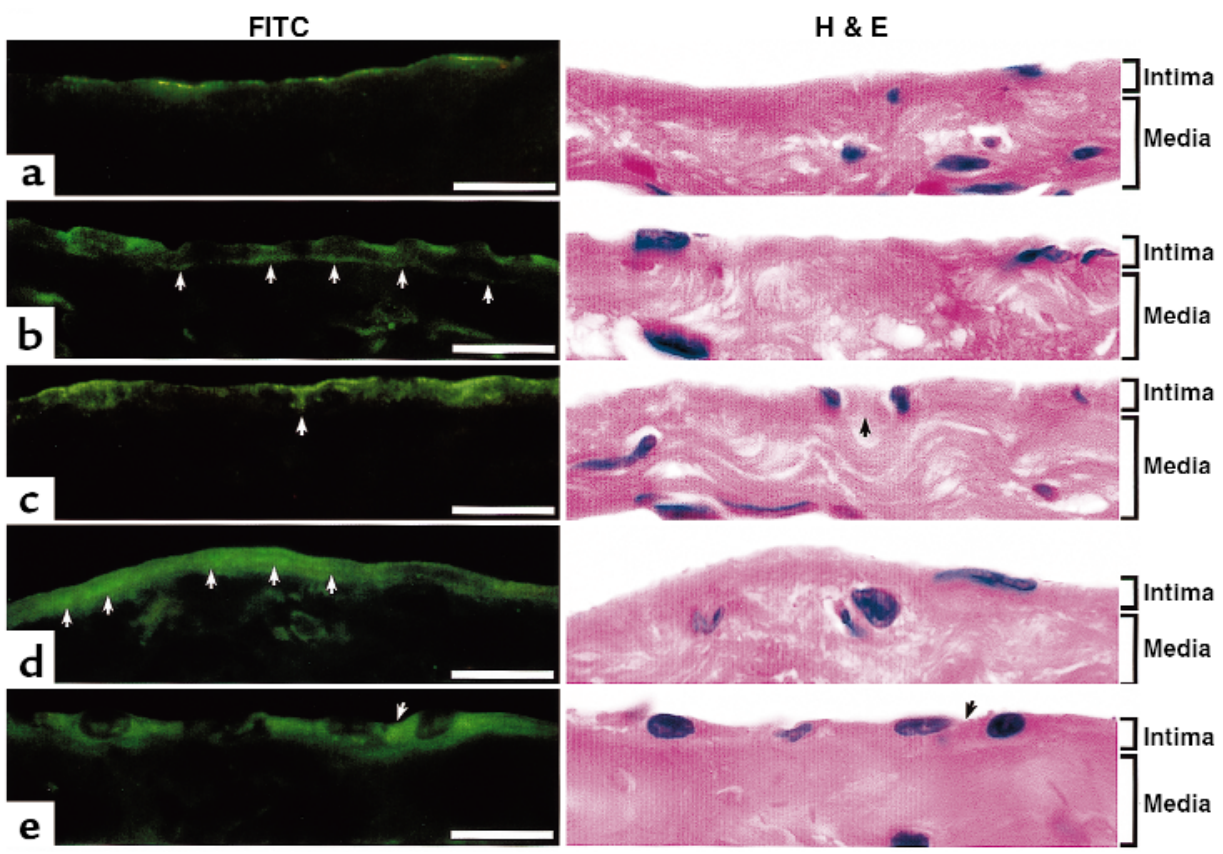

Figure 2

Representative transverse sections of the IVC segment after culturing for 4 hours. Sections in the left panels were reacted with antibodies against PECAM-1 (a), integrin $\alpha v(\mathbf{b})$, VE-cadherin (c), paxillin (d), and Cx43 (e), and stained with FITC. Adjacent sections were stained with $\mathrm{H} \& \mathrm{E}$ (right side). Endothelial cells locate in the intima, and smooth muscle cells locate in the media of IVC. Pericytes may be present in the boundary between the intima and media. $\mathrm{H} \& \mathrm{E}$ stains nuclei of these cells dark blue. Arrows in $\mathbf{b}$ and $\mathbf{d}$ indicate the staining on the endothelial basal membranes. Arrows in $\mathbf{c}$ and $\mathbf{e}$ indicate the staining (left side) at the site of endothelial cell-cell attachment. Bar: $20 \mu \mathrm{m}$.

fin, and cut serially. Sections were incubated with antibodies against $\mathrm{Cx} 26$ (CX-12H10; Zymed), Cx43 (clone 2; Transduction Laboratories, Lexington, Kentucky, USA), VE-cadherin (cadherin-5; clone 75; Transduction Laboratories), paxillin (clone 349; Transduction Laboratories), integrin $\alpha_{\mathrm{v}}$ (H9.2B8; PharMingen, San Diego, California, USA), and PECAM-1 (MEC13.3; PharMingen) at 1:100 dilution overnight at $4^{\circ} \mathrm{C}$. For negative controls, isotype-matched immunoglobulins, mouse IgG1 (MOPC-21; Sigma Chemical Co., St. Louis, Missouri, USA); hamster IgG, group 3 (A19-4; PharMingen), and rat IgG2a (R35-95; PharMingen), were used at the concentration as specific antibodies. The sections were washed with PBS and stained with FITCconjugated secondary antibodies at 1:1000 dilution using anti-mouse IgG (MBL, Nagoya, Japan) for Cx26, Cx43, VE-cadherin, and paxillin; anti-hamster IgG (cocktail; Pharmingen) for integrin $\alpha \mathrm{v}$; and anti-rat IgG (MBL) for PECAM-1. Sections were observed through an Axioplan2 microscope (Carl Zeiss) equipped for epifluorescence. Adjacent sections were stained with hematoxylin and eosin ( $\mathrm{H} \& \mathrm{E})$.

Spontaneous metastasis assay. Cells $\left(2 \times 10^{5}\right)$ were injected subcutaneously into the footpad of a 4-week-old, male C57BL/6 mouse (four mice per clone). Tumor size was monitored by direct measurement of diameters every 2 or 3 days. Three weeks later, when tumors reached a diameter of $8-10 \mathrm{~mm}$, tumor-bearing legs were amputated along with the right popliteal lymph node. The mice were allowed to survive a further 4 weeks; then autopsies were performed and metastatic colonies formed in the bilateral lungs were counted. Independent experiments were repeated three times.

Human melanocytic samples. Melanoma samples were obtained from 12 patients with skin melanoma. The patients underwent surgery at the time of diagnosis and their progress was followed for more than 5 years in the Osaka University Hospital. Five lesions with intradermal nevus were excised from individual patients in the same hospital. Surgical samples were fixed in $4 \%$ paraformaldehyde and paraffin-embedded. Sections were used for diagnosis and immunohistochemistry. Melanoma lesions were diagnosed according to Clark's level (31).

\section{Results}

Cx26-dependent dye coupling between melanoma and endothelial cells. While examining the difference in gene expression levels between F10 and BL 6 cells by the cDNA subtraction method, we found that $\mathrm{Cx} 26$ gene expression was upregulated by fivefold in BL6 cells (Figure 1a). Consistently, BL6 cells expressed a significantly higher level of the Cx26 protein compared with F10 cells (Figure $1 \mathrm{~b})$. In B16, which is the parental strain of both F10 and BL6 cells, the expression level of the Cx26 gene and protein was similar to that of F10 cells (data not shown). The mRNA levels for other connexins were below the limit of detection in both F10 and BL6 cells (Figure 1a). Five clones carrying the full-length Cx26 cDNA were isolated from a BL6 cDNA library and sequenced. No 
mutations were detected in their coding regions. We performed the dye-transfer assay to examine whether GJIC ability increased in BL6 cells. Under in vitro culture conditions, neither F10 nor BL6 cells showed any GJIC ability by themselves (data not shown). We also examined dye transfer from melanoma to NIH3T3 fibroblastic monolayer cells and from melanoma to SVEC4-10 or HUV-EC-C endothelial monolayer cells under in vitro culture conditions. Here again, little or no dye coupling was detected (data not shown).

Hence, we devised a coculture in which we placed an opened vein segment, with its luminal surface uppermost, at the bottom of a culture dish and seeded melanoma cells onto it. We investigated whether opened IVC segments preserved the integrity of the endothelial cell monolayer during cultivation. At the start of cultivation, only the luminal surfaces of the IVC segments located close to the cut edges were stained with trypan blue, but the central regions (above $80 \%$ of the total surface) were not (data not shown). This was the case for IVC segments after 2-6 hours of cultivation (data not shown). To further identify the endothelial cells, transverse sections of the IVC segments were reacted with antibodies against PECAM-1 and integrin $\alpha v$, two proteins specifically expressed on endothelial cell membranes. Both antibodies stained the luminal surface of the IVC segment (Figure 2, a and b). In addition, the reactivity of the anti-integrin $\alpha_{\mathrm{V}}$ antibody drew a line that paralleled the luminal sur- face, corresponding to the endothelial basal membrane (Figure $2 \mathrm{~b}$ ). These results indicated that IVC segments retained a continuous endothelium in their uppermost layers despite the occasional absence of nuclei. The VEcadherin antibody preferentially stained the endothelial luminal membranes; some staining appeared to locate at the site of endothelial cell-cell attachment (Figure 2c). Paxillin staining was detected as punctate inside the endothelial cells and mainly overlapped with integrin $\alpha \mathrm{v}$ staining (Figure $2 \mathrm{~d}$ ). Northern blotting using the IVC segment revealed that the mRNA levels for connexins other than $\mathrm{Cx} 43$ were below the limit of detection (Figure 1a). The Cx43 protein was located widely in the endothelial cytoplasm, but some staining was concentrated at the site of endothelial cell-cell attachment (Figure 2e). The immunoreactivity of endothelial cells did not change after 6 hours of cultivation (data not shown). No specific immunostains were detected in any negative control experiments (data not shown). These results suggested that endothelial cells preserved their integrity with respect to both cellcell and cell-extracellular matrix interactions throughout the culture periods.

When F10 or BL6 cells were seeded onto the opened IVC segments, most melanoma cells settled down on the IVC surface within 2 hours. Consistent with the result of Western blot (Figure 1b), BL6 cells, but not F10 cells, expressed Cx26 during the coculture period (Figure 3, a and b). Some immunostaining was con-

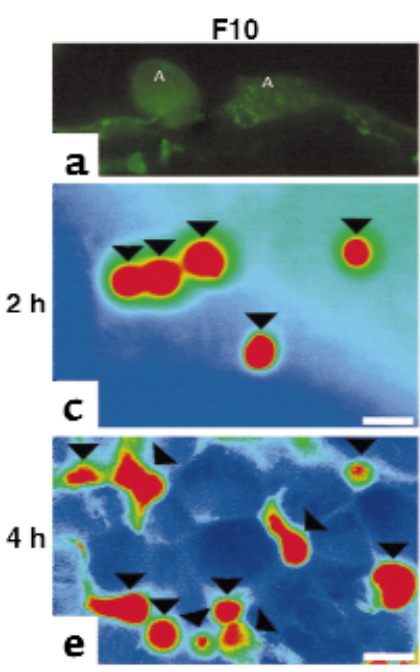

Figure 3

Dye-transfer assay using the coculture composed of melanoma cells and IVC segments. ( $\mathbf{a}$ and $\mathbf{b}$ ) Cx26 expression in melanoma cells after culturing on IVC for 4 hours. Transverse sections of the cocultured tissue containing F10 ( $A$ in panel a) or BL6 (B in panel b) cells were stained with anti-Cx26 antibody. Arrows indicate the localization of Cx26 protein on the cell membrane. Also, note that endothelial cells of IVC contain much less Cx26 than Cx43. Bar: $20 \mu \mathrm{m}$. (c-f) BCECF-labeled F10 (c and $\mathbf{e}$ ) or BL6 (d and f) cells were seeded onto an IVC segment and observed from directly above the coculture at 2 ( $\mathbf{c}$ and $\mathbf{d}$ ) and 4 (e and $\mathbf{f}$ ) hours using a microscope equipped for epifluorescence. The relative intensity of fluorescence is displayed by the 256-color spectrum; red indicates a greater intensity than blue. Arrowheads indicate BCECFlabeled F10 and BL6 cells. Bar: $20 \mu \mathrm{m}$. (g) The number of cells showing dye coupling with endothelial cells was counted in a sample of 500 BCECF-labeled cells observed after 4 hours of coculture. Data are expressed as the mean of three independent experiments. Bars $=\mathrm{SE} ; \mathrm{SE}$ was sometimes too small to be represented. ${ }^{A} P<0.01$ by $t$ test when compared with the value obtained from $\mathrm{F} 10$ cells. ${ }^{B} P<0.01$ by $t$ test when compared with the value obtained from BL6 cells. 


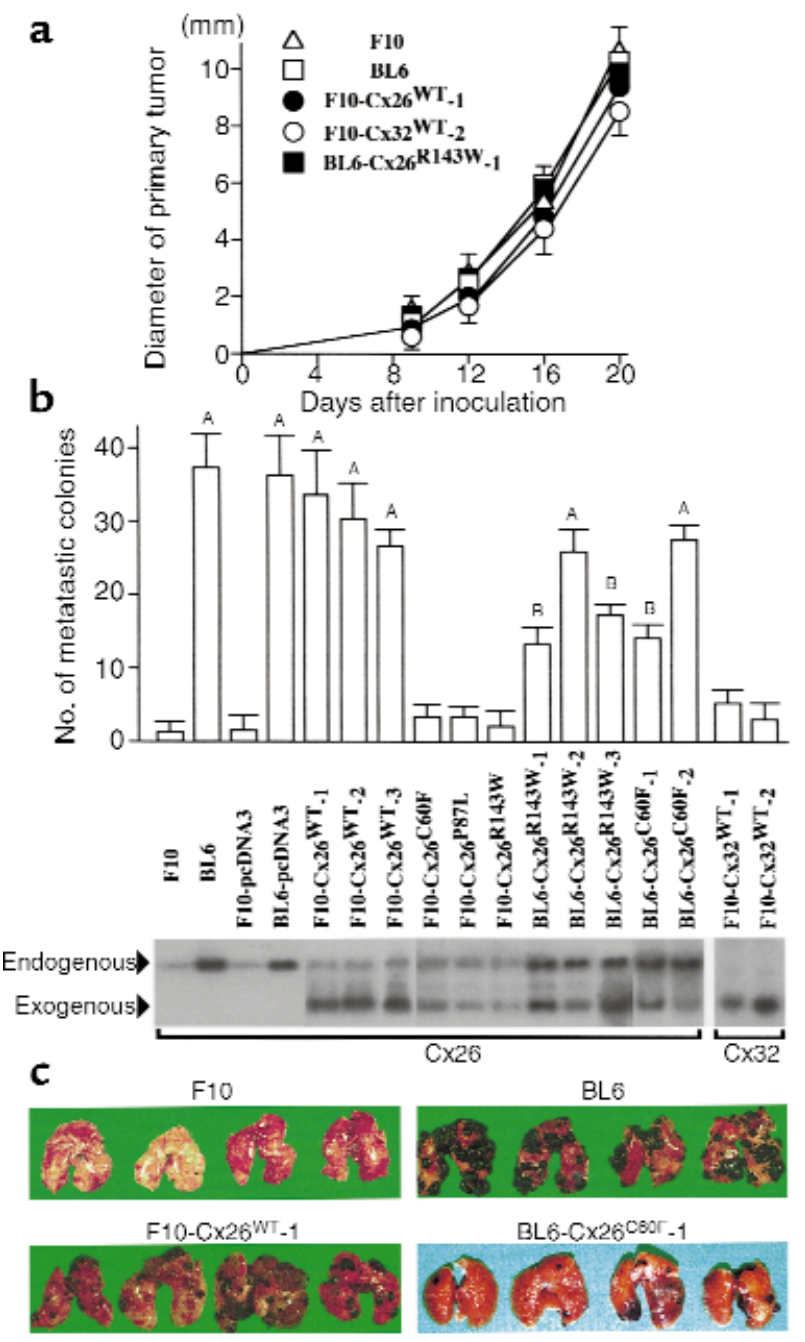

Figure 4

Spontaneous metastasis assay of Cx26 and Cx32 transfectants. (a) Primary tumor growth at the inoculation site (right footpad). Growth curves for the five representative clones are expressed as the mean and $\mathrm{SE}$ of three independent experiments. The values of the other clones used were within the range of these five clones at any time point. There was no significant difference between the clones. (b) The number of colonies in the lungs produced by various $\mathrm{F} 10$ and BL6 clones. F10pcDNA3 and BL6-pcDNA3 clones are vector controls. Data are expressed as the mean and SE of three independent experiments. ${ }^{A} P$ $<0.05$ by $t$ test when compared with the value obtained from F10 cells. ${ }^{\mathrm{B} P}<0.05$ by $t$ test when compared with the value obtained from BL6 cells. The Northern blot shows the detection of endogenous and exogenous Cx26 and Cx32 mRNA in each clone. (c) Representative results of the lung metastatic colonies produced by F10, BL6, F10Cx26 ${ }^{\mathrm{WT}}-1$, and BL6-Cx26 $6^{\mathrm{C} 60 \mathrm{~F}}-1$ cells. Note that BL6 and BL6-Cx26 $660 \mathrm{~F}$ 1 cells produced solid black colonies whereas $\mathrm{F} 10-\mathrm{C} \times 26^{\mathrm{WT}}-1$ cells produced amelanotic colonies as well as melanotic ones.

centrated on the BL6 cell membrane facing the vein (Figure 3b). Next, we seeded BCECF-labeled melanoma cells onto the IVC segment and observed the dye-transfer profile directly above the coculture with a microscope equipped for epifluorescence. At 2 hours of coculture, all the F10 and BL6 cells displayed strong fluorescence in their cytoplasm, demonstrating the absence of dye transfer (Figure 3, c and d). When we scanned the coculture at 4 hours, we occasionally found a weakly fluorescent area around the BL6 cells, but not around the F10 cells (Figure 3e). At higher magnification, dye transfer from BL6 cells into endothelial cells was clearly demonstrated (Figure 3f). The dye was also transferred between neighboring endothelial cells. At 6 hours, most BL 6 cells lost their fluorescence, whereas most F10 cells remained as fluorescent as those at 4 hours (data not shown).

To examine this phenomenon more systematically, we prepared five dishes containing cocultures of F10 or BL6 cells with IVC segments. Each cocultured tissue was fixed with glutaraldehyde every 15 minutes from 3 hours 30 minutes to 4 hours 30 minutes. In each coculture, we examined 500 BCECF-labeled F10 or BL6 cells seeded onto the IVC segment. At 4 hours of coculture, on average, 22 BL6 cells showed dye coupling with endothelial cells (Figure 3g). When we observed the cocultured tissues fixed at other intervals, the number of BL6 cells showing dye coupling with endothelial cells was similar to that at 4 hours (data not shown). In contrast, little or no dye coupling was observed in any F10 cell-containing cocultures.

Various F10 and BL6 clones were obtained by transfection with wild-type and mutant forms of $\mathrm{Cx} 26$ and Cx32 (Figure 4b, blot). The C60F, P87L, and R143W mutations of $\mathrm{Cx} 26$ locate in the first extracellular and second and third transmembrane domains, respectively, and exert different dominant-negative effects on Cx26 (25). The former abrogates Cx26-mediated GJIC and the latter two abrogate $\mathrm{Cx} 26$-mediated cell growth control in HeLa cells (25). Transfectant clones were examined for GJIC ability with endothelial cells according to the protocol above. Figure $3 \mathrm{~g}$ showed the results of the 4-hour coculture; the results at the other four intervals were essentially similar. F10-Cx $26^{\mathrm{WT}}-1,-2$, and -3 cells showed dye coupling as frequently as BL6 cells. Transfection with the C60F or R143W-mutant form did not elevate the dye-coupling ability of F10 cells, whereas transfection with the P87L-mutant form produced a few dye coupling-competent F10 cells. Thus, the former two mutant forms were introduced into BL6 cells to examine whether these mutant forms abrogate the dye-coupling competency of BL6 cells. Among cells of two BL6-Cx26 $6^{\mathrm{C} 60 \mathrm{~F}}$ and three BL6-Cx26 $6^{\mathrm{R} 143 \mathrm{~W}}$ clones, only a few showed dye coupling, indicating a dominant-negative effect of the C60F and R143W mutations in this assay. F10-Cx $32^{\mathrm{WT}}-1$ and -2 cells were deficient in dye coupling.

Metastatic phenotype of Cx transfectants. We examined the metastatic proficiency of dye coupling-competent F10 clones and dye coupling-deficient BL 6 cells. The original melanoma cells and their transfectants were injected subcutaneously into mouse footpads. Tumor growth at the injection sites was the same among all the clones examined (Figure 4a). F10 clones transfected with wild-type Cx26 were more metastatic than untransfected F10 cells, and transfection with mutant 
forms of $\mathrm{Cx} 26$ did not alter the metastatic phenotype of F10 cells (Figure 4b). Some lung colonies produced by F10-Cx $26^{\text {WT }}$ clones looked amelanotic, which contrasted with the solid black appearance of BL6-derived colonies (Figure 4c). C60F and R143W mutations produced an inhibitory effect on the spontaneous metastasis of BL6 cells. Of the five dye coupling-deficient BL6 clones, BL6-Cx $26^{\mathrm{C} 60 \mathrm{~F}}-1$, BL6-Cx $26^{\mathrm{R} 143 \mathrm{~W}}-1$, and -3 cells displayed threefold less metastatic colonies compared with the original BL6 cells (Figure 4, b and c). On the other hand, the reduction in the number of metastatic colonies by other BL6 clones was small. There was the possibility that the in vivo expression levels of $\mathrm{Cx} 26$ differed from that of the in vitro levels. We examined the Cx26 gene expression in the tumors of various clones growing in footpads and lungs and found that the expression level for the endogenous and exogenous $\mathrm{Cx} 26$ gene in each clone was essentially the same as that of cultured cells (data not shown).

Cx26 expression in buman melanoma. The clinical relevance of $\mathrm{Cx} 26$ to malignant phenotypes of human melanocytic lesions was assessed by immunohistochemistry. We examined five cases with intradermal nevus, five cases with in situ melanoma (Clark's level I), and seven cases with more advanced melanoma (Clark's level III or IV). Nevus cells and in situ melanoma cells were $\mathrm{C} \times 26$ negative in all of the cases examined (data not shown and Figure 5, a and b). In the specimen of nevus, we observed the epidermis apart from the nevus lesion and found that melanocytes were also Cx26 negative under physiological conditions (data not shown). In the cases of advanced melanoma, immunoreactivity varied at sites containing melanoma cells. Melanoma cells resident within the epidermal basal layer were Cx26 negative or faintly positive in all seven cases examined (Figure 5, c and d). In contrast, melanoma cells detached from the basal layer or in the process of invading the dermis often showed Cx26-positive staining (Figure 5, $c$ and $d$ ). This increase in the immunoreactivity of melanoma cells was detected in six of the seven cases.

\section{Discussion}

Although Cx expression in melanocytes or melanoma cells has not been studied intensively, we found that BL6 cells expressed the Cx 26 gene as abundantly as liver tissues. Hepatocytes are one of the cell types that express Cx 26 most abundantly and exhibit an efficient dye coupling with one another $(32,33)$. Since there was no mutation in the coding sequence of the $\mathrm{C} x 26$ mRNA expressed in BL6 cells, BL6 cells were expected to be as GJIC proficient as hepatocytes. Unexpectedly, we could detect little dye coupling between BL6 cells in culture. Also, little or no dye transfer was detected from BL6 cells into endothelial cells cultured on dishes. Instead, we detected dye transfer from BL6 cells into endothelial cells making up the IVC. In addition to BL6 cells, F10 cells acquired the similar dye-transfer ability by transfection with Cx26. Transfection with dominant-negative forms of $\mathrm{Cx} 26$ abrogated the dye-cou- pling proficiency of BL6 cells. These results indicated that dye-coupling between melanoma and endothelial cells was a Cx26-specific event. However, the nature of the connexins on endothelial cells that participate in the formation of gap junctions with Cx26-expressing melanoma cells remains unclear. There was little, if any, Cx26 expression in the IVC segments at either the mRNA or protein level (Figure 1a and Figure 3, a and b). It has been shown that a heterotypic gap junction between Cx32 and Cx26 transferred dye as efficiently as a Cx26-homotypic gap junction (34). However, it is not likely that $\mathrm{Cx} 32$ on endothelial cells is a counterpart of Cx26 on melanoma cells, since Cx32-transfected F10 clones were deficient in dye coupling with endothelial cells. Endothelial cells have been known to express several Cx subtypes, including Cx37, Cx40, and Cx43 (35).

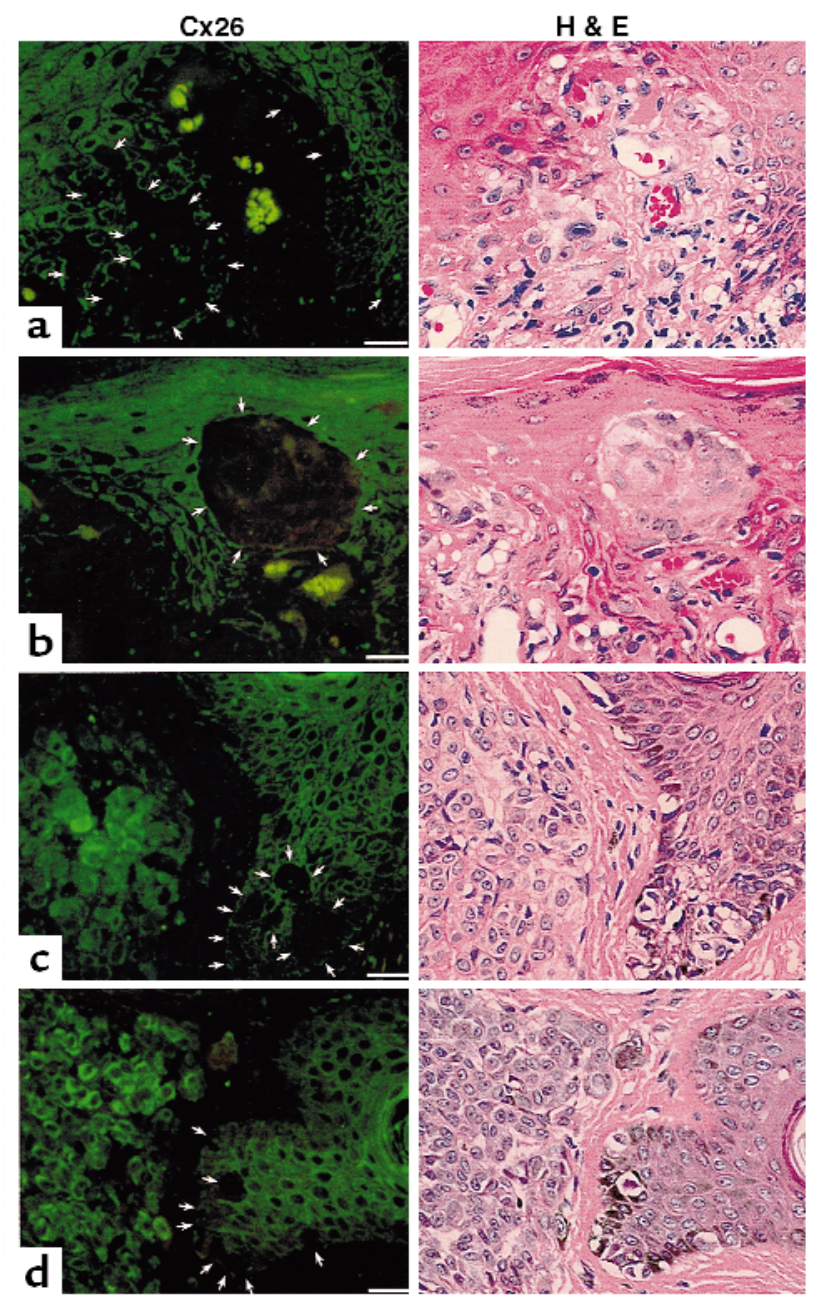

\section{Figure 5}

Histologic examination of human melanoma tissues by staining with anti-Cx26 antibody (left side) and $\mathrm{H} \& \mathrm{E}$ (right side). Four representative cases are shown. ( $\mathbf{a}$ and $\mathbf{b}$ ) Two cases with in situ melanoma (Clark's level $=\mathbf{I})$. $(\mathbf{c}$ and $\mathbf{d})$ Two cases with advanced melanoma (Clark's level = III). Keratinocytes served as an internal control for immunohistochemical staining (21). Arrows indicate melanoma cells or lesions still resident within the epidermis, most of which were $\mathrm{Cx} 26$ negative. Note that the reactivity for $\mathrm{Cx} 26$ antibody increased in melanoma cells invading into the dermis, as seen in the left half of $\mathbf{c}$ and $\mathbf{d}$. Bar: $75 \mu \mathrm{m}$. 
We detected the $\mathrm{Cx} 43$ gene expression in the IVC segments (Figure 1a) and determined the $\mathrm{Cx} 43$ protein localization in endothelial cells of IVC (Figure 2e). However, the $\mathrm{Cx} 26-\mathrm{Cx} 43$ heterologous gap junction is insufficient for communication between Cx-transfected HeLa cells in culture (34). Efficient formation of GJIC requires the integrity of both cell-cell and cell-extracellular matrix interactions (36-38). Since endothelial cells of the IVC segment appeared to preserve this type of integrity (Figure 2) they could communicate with melanoma cells through the $\mathrm{Cx} 26-\mathrm{Cx} 43$ heterologous gap junction.

At most, $5 \%$ of the BL 6 cells seeded onto IVC segments transferred dye into endothelial cells at a given time point. This raises the possibility that only a portion of the BL6 cell population was able to transfer dye. The following results may throw some light on this issue: (a) at 6 hours, most BL6 cells had lost their fluorescence, whereas most F10 cells remained as fluorescent as those at 4 hours; (b) the expression level of the $\mathrm{Cx} 26$ gene in BL6 cells did not change even after their metastasis into the lung; and (c) none of the transfectant clones of BL6 displayed altered expression levels of the endogenous Cx26 gene (Figure $4 \mathrm{~b}$ ). These results do not exclude, but rather favor, the interpretation that BL6 cells were a dye coupling-competent cell population. On the other hand, F10 cells rarely showed dye coupling with endothelial cells at any time point, indicating that F10 cells are a dye coupling-deficient cell population.

We examined whether such a difference in GJIC ability might be involved in metastasis of F10 and BL6 cells. All the three dye coupling-proficient F10 clones, obtained by transfection with wild-type Cx26, were as spontaneously metastatic as BL6 cells. Three out of five dye coupling-deficient BL6 clones, obtained by transfection with dominant-negative forms of $\mathrm{Cx} 26$, significantly reduced their spontaneously metastatic potential. On the other hand, the reduction in the number of metastatic colonies was small in the two BL6 clones of the rest. This may have been due to the lower expression of C60F and R143W dominant-negative forms in these clones in vitro (Figure $4 \mathrm{~b}$, blot). Although there were no direct data to indicate how Cx26 might exert its effects in vivo, the results above suggested an involvement of heterologous gap junction between melanoma and endothelial cells in spontaneous metastasis. F10 cells are as proficient as BL6 for the colonization of the lung after intravenous injection, but deficient after subcutaneous injection (1). The metastatic behavior of BL6 cells appears to differ from that of F10 cells before or during the process of tumor cell intravasation at the primary site. Cx26 may function during the process of intravasation around the primary sites. This is reminiscent of a report that demonstrated GJIC formation between leukocytes and endothelial cells at the site of leukocyte permeabilization of blood vessels (39). Melanoma cells are likely to use the same GJIC-dependent mechanism to enter the blood vessel $(40,41)$. This type of heterologous GJIC might trigger the endothelial cell dissocia- tion at the site of a tumor cell-endothelial cell interaction (42). However, our data could not rule out Cx26 involvement in other stages of metastasis.

Cx26 expression was low not only in melanocytes and nevus cells, but also in melanoma cells that were still resident in the epidermal basal layer. The results were consistent with the fact that $\mathrm{Cx} 26$ has been characterized as a carcinogenesis suppressor $(11,15)$. In contrast, melanoma cells markedly increased their expression level of Cx26 after detachment from the epidermis and in the process of invading the dermis (Figure 5). Kamibayashi et al. (21) reported the decline of Cx26 expression in the course of development of carcinogenesis from papilloma to squamous cell carcinoma, another type of skin tumor originated from keratinocytes. Interestingly, they also demonstrated that Cx26 expression apparently increased in the cancer cells metastasizing into lymph nodes. Their results and ours consistently suggest a possibility that once tumor cells have acquired autonomous growth potential and detached from the primary sites, higher expression of Cx26 may promote invasion and metastasis.

Several recent reports support this speculation. Vaccines prepared from $\mathrm{Cx} 37$-derived peptides have been reported to protect mice from spontaneous metastasis of Lewis lung carcinoma (43). Transfection with Cx43 increased the invasive potential of HeLa cells on heart cells after formation of heterotypic dye coupling between both cells (44). Here, we found a correlation in melanoma cells between their metastatic phenotypes and GJIC proficiency. Malignant glioma ranks with malignant melanoma as a tumor with a very poor prognosis $(45,46)$. Recently, functional gap junctions were found to form between glioma cells and surrounding astrocytes, resulting in the phenotypic transformation of the astrocytes (47). This kind of Cx function might be called "acclimation." Both glioma and melanoma cells are able to communicate directly with distinct types of normal host cells. Glioma cells, characterized by their diffuse infiltration into surrounding brain tissue (45), are coupled with astrocytes. Melanoma cells, characterized by a high incidence of metastasis (46), are coupled with endothelial cells. Such couplings might contribute to the susceptibility of the host to tumor invasion.

\section{Acknowledgments}

We thank Kenji Morihana and Akinori Fukuyama for technical assistance with histologic experiments. This work was supported by a Grant-in-Aid for Scientific Research on Priority Areas from the Ministry of Education, Science, Sports and Culture of Japan and grants from the Osaka Cancer Society, The Naito Foundation, and Seijinbyo-igaku Foundation.

\footnotetext{
Poste, G., Doll, J., Hart, I.R., and Fidler, I.J. 1980. In vivo selection of murine B16 melanoma variants with enhanced tissue-invasive properties. Cancer Res. 40:1636-1644.

2. Kobori, M., et al. 1998. Large scale isolation of osteoclast-specific genes by an improved method involving the preparation of a subtracted cDNA library. Genes Cells. 3:459-475.

3. Ito, A., et al. 1998. Systematic method to obtain novel genes that are reg-
} 
ulated by $m i$ transcription factor: impaired expression of granzyme B and tryptophan hydroxylase in $\mathrm{mi} / \mathrm{mi}$ cultured mast cells. Blood. 91:3210-3221.

4. Ito, A., et al. 1999. Inhibitory effect of the transcription factor encoded by the $m i$ mutant allele in cultured mast cells of mice. Blood. 93:1189-1196.

5. Nakaji, T., et al. 1999. A new member of the GTPase superfamily that is upregulated in highly metastatic cells. Cancer Lett. 147:139-147.

6. Ito, A., et al. 2000. A truncated isoform of the PP2A B56 subunit promotes cell motility through paxillin phosphorylation. EMBO J. 19:562-571.

7. Beyer, E.C., Paul, D.L., and Goodenough, D.A. 1990. Connexin family of gap junction proteins. J. Membr. Biol. 116:187-194.

8. Charles, A.C., et al. 1992. Intercellular calcium signaling via gap junctions in glioma cells. J. Cell Biol. 118:195-201.

9. Reaume, A.G., et al. 1995. Cardiac malformation in neonatal mice lacking connexin43. Science. 267:1831-1834.

10. Kirchhoff, S., et al. 1998. Reduced cardiac conduction velocity and predisposition to arrhythmias in connexin40-deficient mice. Curr. Biol. 8:299-302.

11. Yamasaki, H., Mesnil, M., Omori, Y., Mironov, N., and Krutovskikh, V. 1995. Intercellular communication and carcinogenesis. Mutat. Res. 333:181-188.

12. Eghbali, B., Kessler, J.A., Reid, L.M., Roy, C., and Spray, D.C. 1991. Involvement of gap junctions in tumorigenesis: transfection of tumor cells with connexin 32 cDNA retards growth in vivo. Proc. Natl. Acad. Sci. USA. 88:10701-10705.

13. Hirschi, K.K., Xu, C.-E., Tsukamoto, T., and Sager, R. 1996. Gap junction genes $\mathrm{Cx} 26$ and $\mathrm{Cx} 43$ individually suppress the cancer phenotype of human mammary carcinoma cells and restore differentiation potential. Cell Growth Differ. 7:861-870.

14. Grossman, H.B., Liebert, M., Lee, I.W., and Lee, S.W. 1994. Decreased connexin expression and intercellular communication in human bladder cancer cells. Cancer Res. 54:3062-3065.

15. Mesnil, M., et al. 1995. Negative growth control of HeLa cells by connexin genes: connexin species specificity. Cancer Res. 55:629-639.

16. Zhu, D., Kidder, G.M., Caveney, S., and Naus, C.C.G. 1992. Growth retardation in glioma cells cocultured with cells overexpressing a gap junction protein. Proc. Natl. Acad. Sci. USA. 89:10218-10221.

17. Nicolson, G.L., Dulski, K.M., and Trosko, J.E. 1988. Loss of intercellular junctional communication correlates with metastatic potential in mammary adenocarcinoma cells. Proc. Natl. Acad. Sci. USA. 85:473-476.

18. Nicolson, G.L., et al. 1990. Lack of correlation between intercellular junctional communication, p21rasEJ expression, and spontaneous metastatic properties of rat mammary cells after transfection with c-H-rasEJ or neo genes. Oncogene. 5:747-753.

19. Hamada, J., Takeichi, N., and Kobayashi, H. 1988. Metastatic capacity and intercellular communication between normal cells and metastatic cell clones derived from a rat mammary carcinoma. Cancer Res. 48:5129-5132.

20. Ren, J., Hamada, J., Takeichi, N., Fujikawa, S., and Kobayashi, H. 1990. Ultrastructural differences in junctional intercellular communication between highly and weakly metastatic clones derived from rat mammary carcinoma. Cancer Res. 50:358-362.

21. Kamibayashi, Y., Oyamada, Y., Mori, M., and Oyamada, M. 1995. Aberrant expression of gap junction proteins (connexins) is associated with tumor progression during multistage mouse skin carcinogenesis in vivo. Carcinogenesis. 16:1287-1297.

22. Saitoh, M., Oyamada, M., Oyamada, Y., Kaku, T., and Mori, M. 1997. Changes in the expression of gap junction proteins (connexins) in hamster tongue epithelium during healing and carcinogenesis. Carcinogenesis. 18:1319-1328.

23. Sakamoto, H., Oyamada, M., Enomoto, K., and Mori, M. 1992. Differential changes in expression of gap junction proteins connexin 26 and 32 during hepatocarcinogenesis in rats. Jpn. J. Cancer Res. 83:1210-1215.

24. Matesic, D.F., Rupp, H.L., Bonney, W.J., Ruch, R.J., and Trosko, J.E. 1994. Changes in gap-junction permeability, phosphorylation, and number mediated by phorbol ester and non-phorbol-ester tumor promoters in rat liver epithelial cells. Mol. Carcinog. 10:226-236.

25. Duflot-Dancer, A., Mensil, M., and Yamasaki, H. 1997. Dominant-negative abrogation of connexin-mediated cell growth control by mutant connexin genes. Oncogene. 15:2151-2158.

26. Nishi, M., Kumar, N.M., and Gilula, N.B. 1991. Developmental regulation of gap junction gene expression during mouse embryonic development. Dev. Biol. 146:117-130.

27. Willecke, K., et al. 1991. Mouse connexin37: cloning and functional expression of a gap junction gene highly expressed in lung. J. Cell Biol. 114:1049-1057.

28. Hennemann, H., et al. 1992. Molecular cloning and functional expression of mouse connexin40, a second gap junction gene preferentially expressed in lung. J. Cell Biol. 117:1299-1310.

29. Hertzberg, E.L., and Skibbents, E.L. 1984. A protein homologous to the 27,000 dalton liver gap junction protein is present in a wide variety of species and tissues. Cell. 39:61-69.

30. Pederson, D.C., and Bowyer, D.E. 1985. Endothelial injury and healing in vitro: studies using an organ culture system. Am. J. Pathol. 119:264-272.

31. Clark, W.H., Jr., From, L., Bernardino, E.A., and Mihm, M.C. 1969. The histogenesis and biologic behavior of primary human malignant melanomas of the skin. Cancer Res. 29:705-726.

32. Traub, O.T., et al. 1989. Comparative characterization of the $21-\mathrm{kD}$ and $26-\mathrm{kD}$ gap junction proteins in murine liver and cultured hepatocytes. J. Cell Biol. 108:1039-1051.

33. Zhang, J.-T., and Nicholson, B.J. 1989. Sequence and tissue distribution of a second protein of hepatic gap junction, $\mathrm{Cx} 26$, as deduced from its cDNA. J. Cell Biol. 109:3391-3401.

34. Elfgang, C., et al. 1995. Specific permeability and selective formation of gap junction channels in connexin-transfected HeLa cells. J. Cell Biol. 129:805-817.

35. Van Rijen, H.V.M., et al. 1997. Gap junctions in human umbilical cord endothelial cells contain multiple connexins. Am. J. Physiol. 272:C117-C130.

36. Jongen, W.M.F., et al. 1991. Regulation of connexin 43-mediated gap junctional intercellular communication by $\mathrm{CA}^{+}$in mouse epidermal cells is controlled by E-cadherin. J. Cell Biol. 114:545-555.

37. Meyer, R.A., Laird, D.W., Revel, J.-P., and Johnson, R.G. 1992. Inhibition of gap junction and adherens junction assembly by connexin and ACAM antibodies. J. Cell Biol. 119:179-189.

38. Lampe, P.D., et al. 1998. Cellular interaction of integrin $\alpha 3 \beta 1$ with laminin 5 promotes gap junctional communication. J. Cell Biol. 143:1735-1747.

39. Jara, P.I., Boric, M.P., and Saez, J.C. 1995. Leukocytes express connexin 43 after activation with lipopolysaccharide and appear to form gap junctions with endothelial cells after ischemiareperfusion. Proc. Natl. Acad. Sci. USA. 92:7011-7015.

40. El-Sabban, M.E., and Pauli, B.U. 1991. Cytoplasmic dye transfer between metastatic tumor cells and vascular endothelium. J. Cell Biol. 115:1375-1382.

41. El-Sabban, M.E., and Pauli, B.U. 1994. Adhesion-mediated gap junctional communication between lung-metastatic cancer cells and endothelium. Invasion Metastasis. 14:164-176.

42. Lewalle, J.M., et al. 1997. Alteration of interendothelial adherens junctions following tumor cell-endothelial cell interaction in vitro. Exp. Cell Res. 237:347-356

43. Mandelboim, O., et al. 1995. Regression of established murine carcinoma metastases following vaccination with tumour-associated antigen peptides. Nat. Med. 1:1179-1183.

44. Graeber, S.H., and Hulser, D.F. 1998. Connexin transfection induces invasive properties in HeLa cells. Exp. Cell Res. 243:142-149.

45. Nelson, J.S., et al. 1982. Necrosis as a prognostic criterion in malignant supratentorial, astrocytic gliomas. Cancer. 52:550-554.

46. Breslow, A. 1970. Thickness, cross-sectional areas and depth of invasion in the prognosis of cutaneous melanoma. Ann. Surg. 172:902-908.

47. Zhang, W., et al. 1999. Direct gap junction communication between malignant glioma cells and astrocytes. Cancer Res. 59:1994-2003. 